\title{
Necrose esofágica aguda secundária a endoprótese: relato de caso de primeiro sobrevivente
}

\author{
Acute esophageal necrosis secondary to stent: \\ case report of the first surviving patient
}

\author{
Leandro Ryuchi Iuamoto ${ }^{1}$, Fábio Hideki Chikami ${ }^{1}$, Ernesto Sasaki Imakuma ${ }^{2}$, \\ Francisco de Salles Collet e Silva ${ }^{3}$
}

Iuamoto LR, Chikami FH, Imakuma ES, Collet e Silva FS. Necrose esofágica aguda secundária a endoprótese: relato de caso de primeiro sobrevivente / Acute esophageal necrosis secondary to stent: case report of the first surviving patient. Rev Med (São Paulo). 2015 jan.-mar.;94(1):29-35.

\begin{abstract}
RESUMO: A necrose esofágica aguda é uma das mais graves complicações associadas ao tratamento endovascular da aorta descendente com colocação de endoprótese. Sua ocorrência é rara, com apenas 3 casos descritos na literatura, entretanto em todos a complicação mostrou-se fatal. O presente trabalho descreve, até onde se sabe, o primeiro caso de necrose esofágica aguda secundária a endoprótese de aorta na qual o paciente sobrevive. Paciente masculino de 53 anos com dissecção de aorta, de artéria subclávia esquerda até bifurcação de artérias ilíacas, realizou tratamento endovascular de colocação de endoprótese. Evoluiu com lesão isquêmica de esôfago médio e distal, para a qual foi indicada esofagectomia total. Após um ano e dois meses, foi submetido a esofagogastroplastia para reconstrução do trato gastrointestinal, com transposição de tubo gástrico retroesternal, a partir da qual permanece praticamente oligossintomático. $\mathrm{O}$ presente trabalho consiste no primeiro relato de caso na qual o paciente sobreviveu à necrose esofágica aguda secundária à colocação de endoprótese de aorta torácica.
\end{abstract}

DESCRITORES: Necrose; Esôfago; Stents; Aorta torácica; Dissecação.

\begin{abstract}
Acute esophageal necrosis is one of the most serious complications associated with endovascular treatment of the descending aorta with stent placement. Its occurrence is rare, with only three cases reported in the literature. However, all this complication proved fatal in all cases. This paper describes, as far as we know, the first case of acute esophageal necrosis secondary to aortic stent graft in which the patient survives. 53 year old male patient with aortic dissection from the left subclavian artery to the bifurcation of the iliac arteries. Patient was submitted to endovascular stent treatment. The patient evolved with ischemic lesion of the middle and distal esophagus and total esophagectomy with gastrostomy and esophagostomy was performed for treatment. After a year and two months, patient underwent esophagogastroplasty for reconstruction of the gastrointestinal tract, with transposition of retrosternal gastric tube, from which remains virtually asymptomatic. The present case report is the first one in which the patient survived a esophagus necrosis secondary to endovascular stent treatment for thoracic aorta dissection.
\end{abstract}

KEYWORDS: Necrosis; Esophagus; Stents; Aorta, thoracic; Dissection.

$2^{\circ}$ lugar Prêmio Oswaldo Cruz no 33 COMU - Congresso Médico Universitário da FMUSP, SP, 31 de out. a 02 de nov. de 2014.

1. Acadêmico do Curso de Medicina da Faculdade de Medicina da Universidade de São Paulo. E-mail: leandro.iuamoto@gmail.com, fabiaohideki@gmail.com.

2. Médico Residente do Hospital das Clínicas da Faculdade de Medicina da Universidade de São Paulo (HC-FMUSP). E-mail: eimakuma@gmail.com.

3. Médico-Doutor Assistente da Disciplina da Cirurgia do Trauma do Hospital das Clínicas da Faculdade de Medicina da Universidade de São Paulo (orientador). E-mail: francisco.collet@hc.fm.usp.br, fscollet@hotmail.com.

Endereço para correspondência: Rua Corveta Camacuã, 391 - Vila Inah. São Paulo, SP. CEP: 05619-020. 


\section{INTRODUÇÃO}

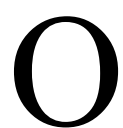

tratamento endovascular da aorta descendente com colocação de endoprótese simplificou consideravelmente o tratamento de doenças da aorta descendente, contribuindo para redução da morbimortalidade pós-operatória em comparação à cirurgia convencional. No entanto, uma das mais graves complicações associadas a esse tratamento é a necrose esofágica aguda devido a obliteração dos vasos responsáveis pela irrigação deste órgão. Trata-se de uma doença rara e pouco descrita na literatura médica, caracterizada por lesão escurecida, de distribuição circunferencial com término abrupto na junção esofagogástrica, presença ou não de exsudato, acometimento geralmente de esôfago distal e ausência de ingestão cáustica. A suspeita da necrose advém de sintomas como: hematêmese, disfagia, hemorragia digestiva alta, melena, vômitos, náuseas e epigastralgia. Complicações como formação de estenoses, fístulas e mediastinites são achados comuns. A investigação inicial envolve a realização de endoscopia digestiva alta e tomografia computadorizada, complementados por biópsia. Histologicamente, a necrose envolve as camadas mucosa e submucosa, na ausência de lesões cáusticas ou por outros agentes. A detecção precoce da necrose esofágica aguda raramente ocorre por se tratar de uma complicação pouco frequente, de instauração rápida, geralmente diagnosticada após sintomas de sangramento em trato digestivo.

Apenas três casos foram descritos na literatura até a data deste estudo e em todos a complicação se mostrou fatal $^{1}$. O presente trabalho descreve, até onde os autores conhecem, o primeiro caso de necrose esofágica aguda secundária a endoprótese de aorta, com sobrevivência do paciente.

\section{RELATO DE CASO}

Paciente masculino de 53 anos, história de dor torácica em dorso, associada a parestesia, paresia e dor em membro inferior esquerdo há 3 dias, procurou o serviço de pronto-socorro de nossa instituição. Como antecedentes pessoais, apresentava hipertensão arterial sistêmica, sem outras comorbidades. Relatou internação hospitalar no início de 2009 por fratura dos dois pés após acidente doméstico, sem cirurgias prévias. Negou tabagismo ou consumo alcoólico. Ao exame físico, paciente apresentava-se com frequência cardíaca de 92 batimentos por minuto, pressão arterial de 230x130 mmHg e saturação de oxigênio igual a $96 \%$, extremidade inferior esquerda fria e pálida, com ausência de pulsos e membro inferior direito perfundido, mas com pulsos das artérias pediosa e poplítea ausentes. Como tratamento inicial foram administrados metoprolol e nitroprussiato de sódio. Foi realizada angiotomografia computadorizada, na qual foi visualizada a dissecção da aorta na altura da artéria subclávia esquerda até a bifurcação das artérias ilíacas (Figura 1).

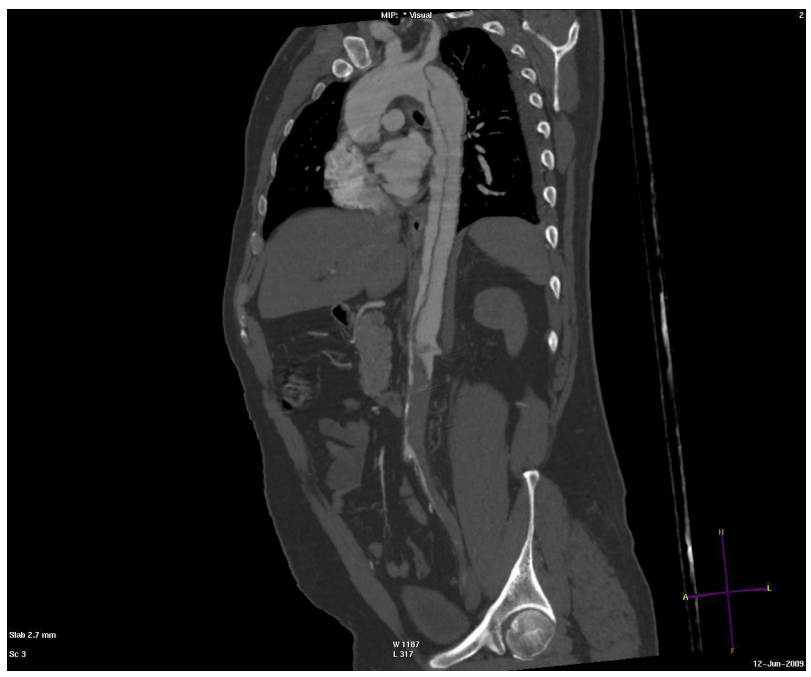

Figura 1. Tomografia Computadorizada - Dissecção iniciada adjacente à emergência da artéria subclávia esquerda no início do segmento descendente da aorta que foi todo acometido e se continua pela bifurcação da artéria ilíaca comum e pela artéria ilíaca esquerda

Foi diagnosticada dissecção aguda de aorta tipo B, sendo realizada cirurgia de emergência endovascular com colocação de endoprótese torácica $34 \times 150$, cobertura da artéria subclávia esquerda, endoprótese $22 \times 80$ na aorta infra-renal e angioplastia com stent $8 \times 37$ e $8 \times 81$ das artérias ilíacas comum e externa esquerda (Medtronic Vascular, Santa Rosa, CA), além de arterioplastia femoral com prótese de pericárdio bovino (Figura 2).

Paciente seguiu em pós-operatório (PO) na UTI, onde evoluiu com desmame de nitroprussiato suspenso no quarto dia de PO, permanecendo hemodinamicamente estável sem necessidade de drogas vasoativas ou ventilação mecânica. Concomitantemente, evoluiu com insuficiência cardíaca, disfunção de ventrículo esquerdo visualizada em ecocardiograma e insuficiência renal aguda com necessidade de diálise. No sexto pós-operatório, apresentou importante quadro de hematêmese, para qual foi realizada endoscopia digestiva alta, que permitiu a visualização das seguintes alterações: 1. Lesão isquêmica de esôfago médio e distal; 2. Pangastrite erosiva hemorrágica; 3. Úlcera bulbar associada à duodenite erosiva; 4. Lesão de Dieulafoy com sinais de sangramento recente em bulbo duodenal.

Paciente foi imediatamente encaminhado à cirurgia, submetido à toracotomia direita com visualização de esôfago de aspecto isquêmico em porção terminal. Realizada esofagectomia total, traqueostomia, gastrostomia e esofagostomia, sendo deixado dreno pleural torácico à direita (Figura 3). Paciente seguiu 
novamente em pós-operatório na UTI, evoluindo com estabilidade hemodinâmica e sem necessidade de drogas vasoativas desde primeiro dia pós-operatório, iniciado piperacilina-tazobactam, com necessidade de ventilação

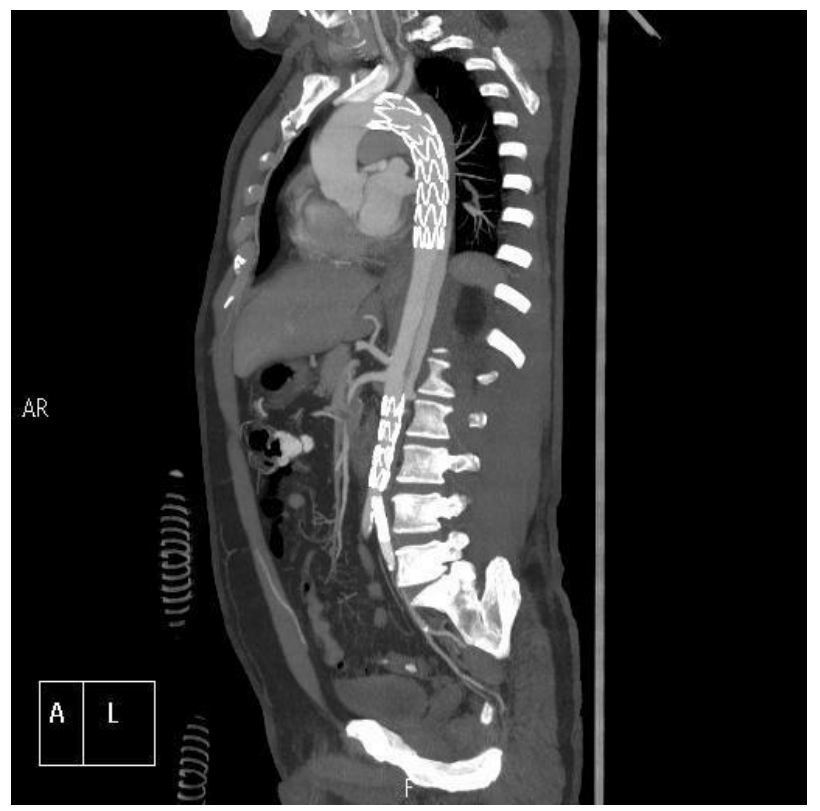

mecânica, mantendo-se em diálise. Após décimo PO, paciente foi extubado, permanecendo confortável em ar ambiente e recebeu alta de unidade de terapia intensiva no décimo quinto pós-operatório de esofagectomia.

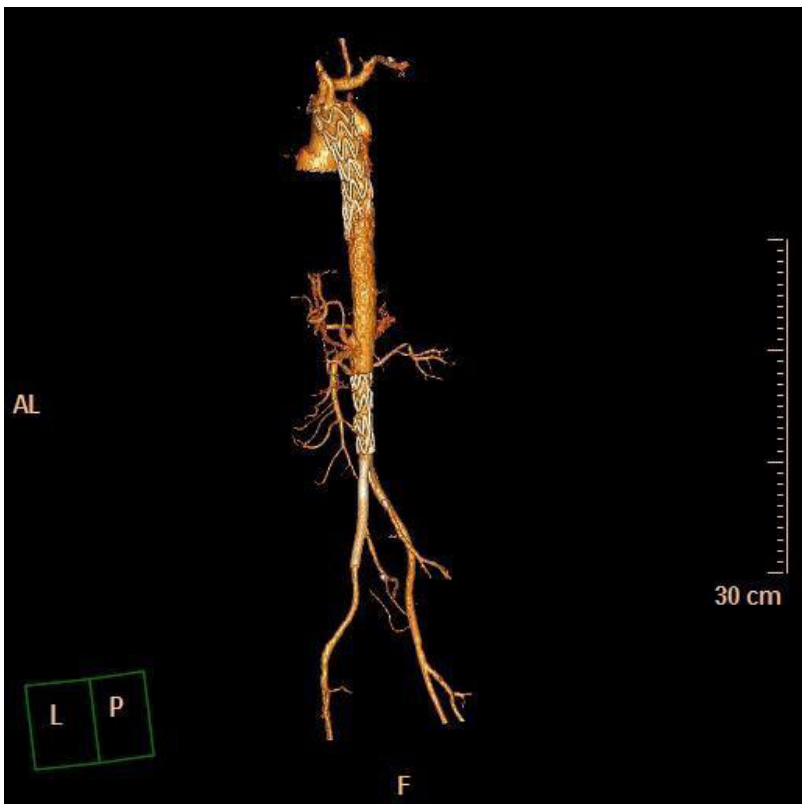

Figura 2. A) Tomografia em corte sagital evidenciando endoprótese que se inicia antes da emergência da artéria subclávia esquerda e se estende até transição tóraco-abdominal. A segunda prótese se inicia imediatamente abaixo das artérias renais até bifurcação das artérias ilíacas. Por fim, a terceira prótese na artéria ilíaca comum esquerda. B) Reconstrução em três dimensões da artéria aorta abdominal e ramos demonstrando a presença das próteses endovasculares

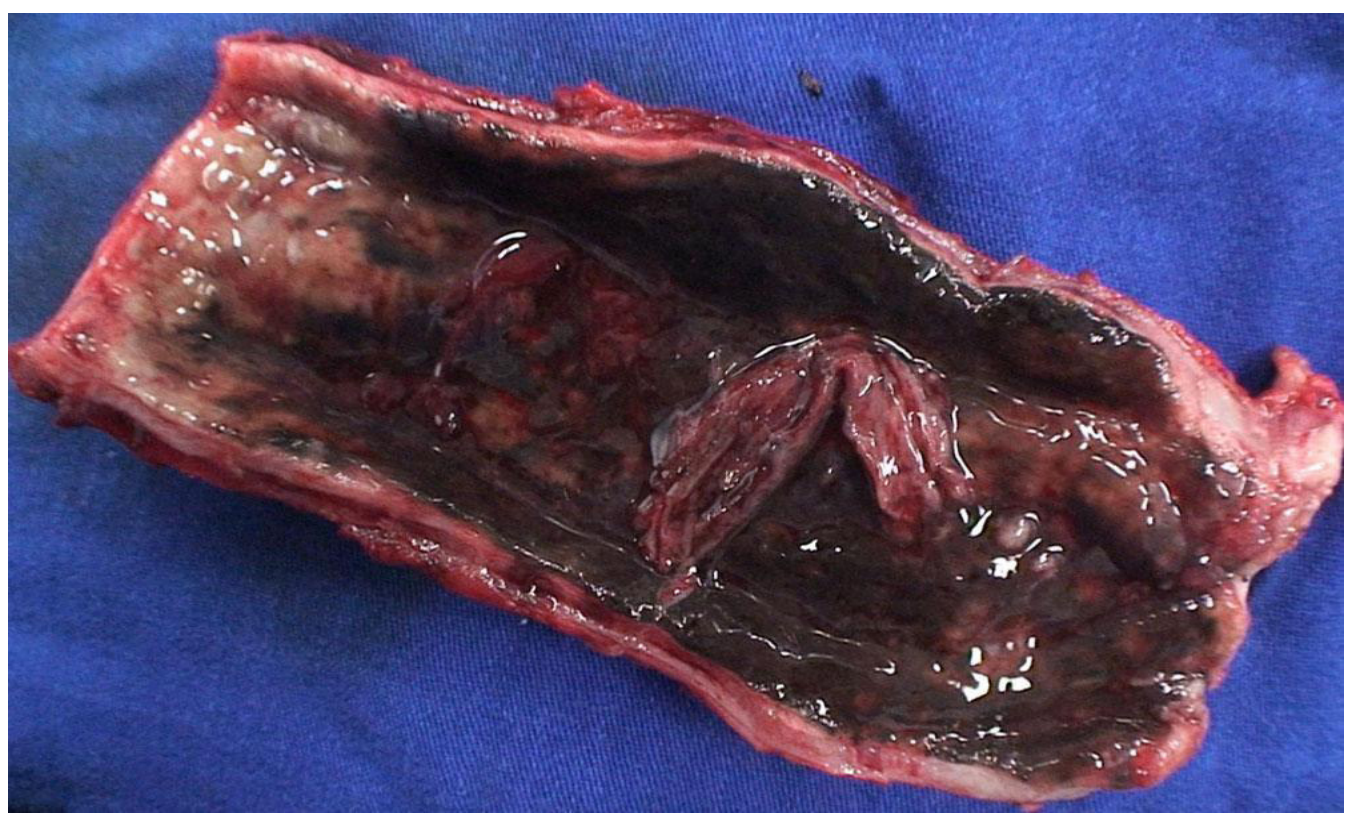

Figura 3. Segmento de esôfago produto de esofagectomia total. A mucosa apresenta perda de pregueamento e coloração castanhaenegrecida com áreas friáveis, o que demonstra necrose de coagulação e ulceração em toda a sua extensão com trombos de fibrina em microvasculatura da submucosa. Tratam-se de características compatíveis com esofagite isquêmica aguda

Logo depois, seu quadro evoluiu para insuficiência renal crônica dialítica, sem perda de peso importante, com alimentação pela gastrostomia, recebeu alta hospitalar no $25^{\circ}$ PO. Foi internado após um ano e dois meses para reconstrução do trato gastrointestinal com realização de esofagogastroplastia com transposição de tubo gástrico retroesternal e anastomose esôfago-gástrica cervical com grampeamento látero-lateral e fechamento de 
traqueostomia.Pacienteevoluiusemsintomasrespiratórios, com alimentação e evacuação normais. $\mathrm{O}$ único sintoma relatado atualmente pelo paciente é a sensação de gosto amargo na boca após jejum prolongado.

\section{DISCUSSÃO}

A etiologia da necrose de esôfago é multifatorial. As principais etiologias consideradas como causas de necrose esofágica aguda são: refluxo ácido e hipoperfusão, obstrução (transitória ou completa) da saída do estômago e hipoperfusão por obstrução de fluxo arterial ${ }^{1,26,32}$. Em relação a esta última etiologia, três fatores podem estar envolvidos no processo de necrose esofágica: 1 . Oclusão arterial aguda secundária ao tratamento endovascular por endoprótese, descrita em pacientes diagnosticados com dissecção aguda de aorta; 2. Compressão mediastinal por hematoma após ruptura de aneurisma; 3. Compressão direta do esôfago por aneurisma ${ }^{1,17,19}$. São raros os casos em que a colocação de endoprótese aórtica gera necrose aguda do esôfago. Na literatura, ocorreram três casos relatados até hoje e o caso estudado seria o quarto ${ }^{1}$. Neste estudo, a prótese utilizada se inicia antes da emergência da artéria subclávia esquerda e se estende até a transição tóraco-abdominal, provavelmente comprometendo grande parte da irrigação sanguínea e acarretando na necrose esofágica em terço médio-distal, compatível com a região mais acometida por isquemia descrita em literatura. Outro mecanismo potencialmente envolvido com a isquemia é a erosão da parede esofágica na região da endoprótese, à semelhança de uma compressão direta por um aneurisma de aorta, acarretando em prejuízo funcional da rede arteriovenosa intramural do esôfago ${ }^{9}$. Esta complementa o sistema de irrigação arterial esofágica, e contribui no estabelecimento de uma rica vascularização, fato que explica a baixa frequência com que a necrose aguda do esôfago ocorre.

O suprimento sanguíneo do esôfago difere dos outros órgãos do tubo digestivo e pode ser dividido em três porções: uma porção cervical, irrigada pela artéria tireóidea inferior e complementada ocasionalmente por ramos da artéria subclávia, carótida comum ou artéria tireóidea superior ${ }^{6}$; Uma porção intratorácica, irrigada por pequenos ramos diretos da aorta descendente, terceira ou quarta artéria intercostal e ramos esofágicos das artérias bronquiais e traqueais; e uma terceira porção abdominal, que é irrigada pela artéria diafragmática inferior esquerda e por ramos da artéria gástrica esquerda ${ }^{10}$. Um suprimento adicional pode ser proveniente de ramos da aorta, artéria esplênica, tronco celíaco e de uma artéria hepática esquerda aberrante $^{1}$. Complementando o suprimento sanguíneo descrito, uma rede vascular presente na camada submucosa integra as vias de aporte sanguíneo. Evidências sugerem que a redução temporária do fluxo sanguíneo esofágico com redução da perfusão deste órgão pode levar à necrose sobretudo da porção abdominal, possivelmente por ser a região menos vascularizada. Nesse contexto, o terço distal do esôfago é o mais propenso a sofrer isquemia. Uma possível explicação para a raridade da necrose esofágica consiste no fato de o suprimento sanguíneo não ser caracterizado por um único tronco arterial de grande calibre, mas sim por múltiplas pequenas tributárias segmentares. Estas, por sua vez, estão associadas à rede arteriovenosa intramural, o que dificulta a obstrução e a falha de aporte sanguíneo a este órgão, sendo necessário um fator obstrutivo importante para gerar hipoperfusão orgânica, como uma prótese endovascular de aorta.

O quadro clínico da necrose aguda de esôfago não é rico em sintomas. Resume-se a hematêmese, melena, epigastralgia e disfagia. No exame físico, podem ser observadas alterações como febre, taquicardia, hipotensão e palidez. Na ausência de hemorragia digestiva alta, nem sempre presente nos casos de necrose do esôfago, o diagnóstico precoce da necrose dificilmente acontece. Isso leva a complicações de elevada mortalidade como fístulas esofágicas, sangramentos maciços, mediastinite e sepse. De acordo com a literatura, o diagnóstico tardio possivelmente explica a mortalidade de $100 \%$ desta patologia ${ }^{1}$. O melhor prognóstico do caso descrito provavelmente se deve à exteriorização precoce da hemorragia, quadro por sua vez possivelmente relacionado à ocorrência de sangramentos importantes em dois pontos diferentes do trato digestivo alto: um no terço médio-distal do esôfago e outro em lesão de Dieulafoy em bulbo duodenal.

Nos casos de suspeita de necrose aguda de esôfago, o exame diagnóstico de escolha é a endoscopia digestiva alta. Os achados endoscópicos são o escurecimento difuso da mucosa esofágica, de aspecto friável, com tecido hemorrágico mais profundo, acometimento segmentar ou completo, mas preferencialmente distal, com término da lesão geralmente na transição gastresofágica, como pode ser visto na Figura $3^{22}$. Possíveis achados endoscópicos associados incluem: úlcera duodenal péptica, erosões e edema de mucosa, sinais de obstrução da saída gástrica ${ }^{6}$, sinais de sangramentos, coágulos e sangue digerido no estômago $^{22}$. A biópsia para confirmação diagnóstica não é necessária. Caso seja realizada, a análise histológica revelará descamação generalizada da mucosa com presença de debris necróticos e epitélio sem viabilidade, eventual necrose da submucosa residual e extenso edema submucoso com intenso infiltrado inflamatório, por vezes estendendo-se até a camada muscular própria observando-se a presença de trombos vasculares, fato que sugere acometimento transmural, como pode ser visto na Figura 4. Presença de ácido periódico de Schiff (com e sem diástase positivo e ferro negativo) sugerem que o pigmento é a lipofucsina que, associado a achados histológicos de necrose e ulceração, confirmam necrose aguda de esôfago ${ }^{6}$. Outro exame que pode dar indícios desta patologia é a tomografia computadorizada de tórax e abdome. Espessamento de esôfago distal e distensão de estômago são achados de necrose aguda esofágica. A tomografia também permite a visualização de alterações 
relacionadas com a etiologia, como aneurisma de aorta e hematoma mediastinal com compressão do esôfago. $\mathrm{O}$ exame complementar permite também o diagnóstico de sinais de complicações da necrose, como pneumomediastino e derrame pleural, indicativos de perfuração da parede esofágica.

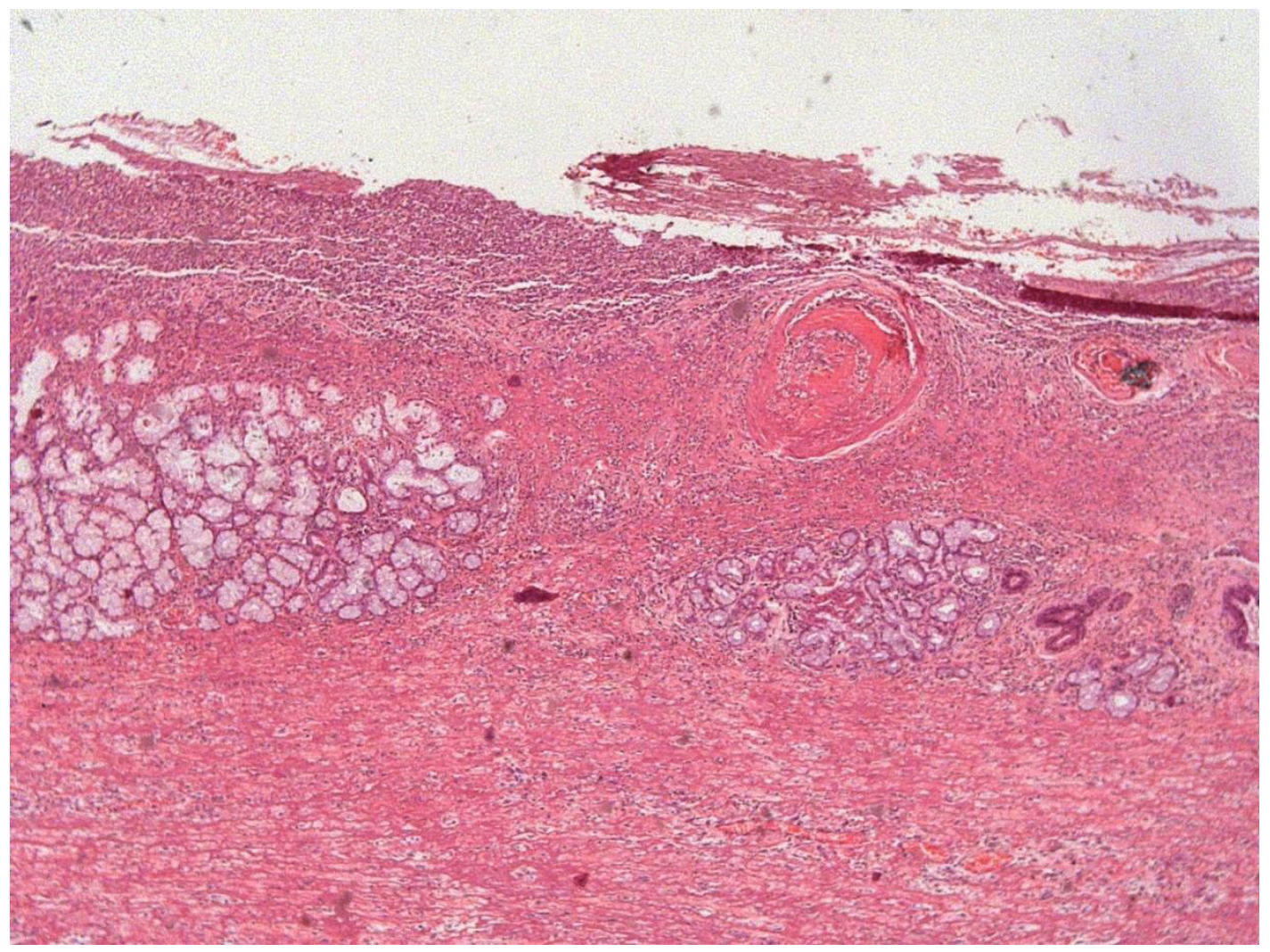

Figura 4: Análise histológica - Descamação generalizada da mucosa com presença de debris necróticos e epitélio sem viabilidade, eventual necrose da submucosa residual e extenso edema submucoso com intenso infiltrado inflamatório, por vezes estendendo-se até a camada muscular própria observando-se a presença de trombos vasculares, fato que sugere acometimento transmural

O tratamento da necrose esofágica aguda é inicialmente de suporte clínico. Consiste na manutenção da estabilidade hemodinâmica, atenção à volemia com hidratação ampla e redução de exposição ácida com inclusão de bloqueadores dos receptores $\mathrm{H} 2$ ou inibidores da bomba de prótons por via endovenosa. É recomendada nutrição parenteral de curto prazo. A descompressão nasogástrica pode ser necessária na persistência de êmese, obstrução da saída gástrica e sangramento ${ }^{33}$. O uso de sucralfato é recomendado devido à sua capacidade citoprotetora e muco excretora ${ }^{13,22}$. Antimicrobianos são indicados em situações de culturas positivas de bactérias e fungos, visualização de células gigantes multinucleadas ou corpos de inclusão na análise histológica por biópsia ${ }^{6}$, em estados de imunossupressão ou em casos de perfuração esofágica. Em casos de úlcera hemorrágica no esôfago, a realização de endoscopia é urgente, havendo necessidade de re-endoscopia para avaliar sua evolução ${ }^{13,22}$. Após a estabilização, o tratamento cirúrgico deve ser realizado. Situações que exigem abordagem cirúrgica precoce são a presença de mediastinite, abscesso, perfuração esofágica, volvo gástrico e associação com trauma de aorta torácica. A cirurgia consiste na ressecção parcial ou total do esôfago. Após a esofagectomia, recomenda-se a ostomia para posterior programação de reconstrução em segundo tempo. Se a condição do paciente permitir e não houver sinais de mediastinite, a reconstrução imediata pode ser aventada. No presente caso, optou-se por realização de ostomia e reconstrução em segundo tempo. A escolha foi adequada para este paciente, uma vez que este sobreviveu ao tratamento, sendo ele o único sobrevivente descrito na literatura para necrose esofágica aguda secundária à colocação de endoprótese aórtica. Após reconstrução do trato alimentar, o paciente permanece oligossintomático.

\section{CONCLUSÃO}

A necrose esofágica aguda é uma complicação rara e de alta morbimortalidade. Em ocorrência secundária à colocação de endoprótese de aorta torácica, eventos 
isquêmicos comprometem a integridade da parede esofágica, cuja mortalidade se aproxima de $100 \%$. O diagnóstico é geralmente tardio e a endoscopia é o exame indicado na investigação da suspeita. A detecção precoce associada a tratamento cirúrgico podem tratar

\section{REFERÊNCIA}

1. De Praetere H, Lerut P, Johan M, Daenens K, Houthoofd S, Fourneau I, Maleux G, Lerut T, Nevelsteen A. Esophageal necrosis after endoprosthesis for ruptured thoracoabdominal aneurysm type I: can long-segment stent grafting of the thoracoabdominal aorta induce transmural necrosis? Ann Vasc Surg. 2010;24(8):1137.e7-12. doi: 10.1016/j. avsg.2010.04.007.

2. Park NH, Kim JH, Choi DY, Choi SY, Park CK, Lee KS, Han SW, Yoo YS. Ischemic esophageal necrosis secondary to traumatic aortic transection. Ann Thorac Surg. 2004;78(6):2175-8. doi: 10.1016/j.athoracsur.2003.07.003.

3. Odelowo OO, Hassan M, Nidiry JJ, Marshalleck JJ. Acute necrotizing esophagitis: a case report. J Natl Med Assoc. 2002;94(8):735-7. Available from: http://www.ncbi.nlm. nih.gov/pmc/articles/PMC2594264/pdf/jnma00325-0099. pdf.

4. Altenburger DL, Wagner AS, Li S, Garavaglia J. A case of black esophagus with histopathologic description and characterization. Arch Pathol Lab Med. 2011;135(6):797-8. doi: 10.1043/2010-0128-C.1.

5. Garas G, Wou C, Sawyer J, Amygdalos I, Gould S. Acute oesophageal necrosis syndrome. BMJ Case Rep. 2011;pii: bcr1020103423. doi: 10.1136/bcr.10.2010.3423.

6. Gurvits GE, Shapsis A, Lau N, Gualtieri N, Robilotti JG. Acute esophageal necrosis: a rare syndrome. J Gastroenterol. 2007;42(1):29-38. doi: 10.1136/bcr.10.2010.3423.

7. Grudell ABM, Mueller PS, Viggiano TR. Black esophagus: report of six cases and review of the literature, 1963-2003. Dis Esophagus. 2006;19(2):105-10. doi: 10.1111/j.14422050.2006.00549.x.

8. Jawadi N, Bisdas T, Torsello G, Stavroulakis K, Donas KP. Endovascular treatment of isolated abdominal aortic dissections: long-term results. J Endovasc Ther. 2014;21(2):324-8. doi: 10.1583/13-4467MR.1.

9. Porcu P, Chavanon O, Sessa C, Thony F, Aubert A, Blin D. Esophageal fistula after endovascular treatment in a type B aortic dissection of the descending thoracic aorta. J Vasc Surg. 2005;41(4):708-11. doi: 10.1016/j.jvs.2004.12.052

10. Swigart LL, Siekert RG, Hambley WC, Anson BJ. The esophageal arteries: an anatomic study of 150 specimens. Surg Gynecol Obstet. 1950;90:234-43.

11. Shek JL, Prietto CA, Tuttle WM, O'Brien EJ. An experimental study of the blood supply of the esophagus and its relation to esophageal resection and anastomoses. $\mathrm{J}$ Thorac Cardiovasc Surg. 1950;19:523-33. efetivamente esta complicação. $O$ presente trabalho consiste no primeiro relato de caso na qual o paciente sobreviveu à necrose esofágica aguda secundária à colocação de endoprótese de aorta torácica.
12. Williams DB, Payne WS. Observations on esophageal bloodsupply. Mayo Clin Proc. 1982;57:448-53.

13. Day A, Sayegh M. Acute oesophageal necrosis: a case report and review of the literature. Int J Surg. 2010;8(1):6-14. doi: 10.1016/j.ijsu.2009.09.014.

14. Kaneda T, Onoe M, Asai T, Mohri Y, Saga T. Delayed esophageal necrosis and perforation secondary to thoracic aortic rupture: a case report and review of the literature. Thorac Cardiovasc Surg. 2005;53:380-382. doi: $10.1055 / \mathrm{s}-2005-865721$

15. Park NH, Kim JH, Choi SY, Park CK, Lee KS, Han SW, Yoo YS. Ischemic esophageal necrosis secondary to traumatic aortic transection. Ann Thorac Surg. 2004;78:2175-2178. doi: 10.1016/j.athoracsur.2003.07.003.

16. Minatoya K, Okita Y, Tagusari O, et al. Transmural necrosis of the esophagus secondary to acute aortic dissection. Ann Thorac Surg 2000;69:1584-1586. doi: 10.1016/ S0003-4975(00)01183-8

17. Watanabe S, Nagashima R, Shimazaki Y, et al. Esophageal necrosis and bleeding gastric ulcer secondary to ruptured thoracic aortic aneurysm. Gastrointest Endosc. 1999;50:847-9.

18. Lee KR, Stark E, Sjaw FE. Esophageal infarction complicatingspontaneousrupture ofthethoracicaorta.JAMA. 1977;237:1233-4. doi:10.1001/jama.1977.03270390049026.

19. Lacombe M, Coquillaud JP, Andreassian B, Baumann J. Acute traumatic rupture of the thoracic aorta with secondary necrosis of the esophagus. Ann Thorac Surg. 1971;11:171-5. doi:10.1016/S0003-4975(10)65434-3.

20. Porcu P, Chavanon O, Sessa C, et al. Esophageal fistula after endovascular treatment in a type B aortic dissection of the descending thoracic aorta. J Vasc Surg. 2005;41:708-11. doi:10.1016/j.jvs.2004.12.052.

21. Rascanu C, Weis-Muller BT, Fürst G, Grotemeyer D, Sandmann W. Esophageal necrosis following endovascular treatment of a ruptured thoracal aortic aneurism: caused by mediastinal compartment syndrome. Chirurgie. 2009;80(544):546-8. doi: 10.1007/s00104-008-1602-y.

22. Lacy BE, Toor A, Bensen SP, Rothstein RI, Maheshwari Y. Acute esophageal necrosis: report of two cases and a review of the literature. Gastrointest Endosc. 1999;49:527-32.

23. McManus JPA, Webb JN. A yeast-like infection of the esophagus caused by lactobacillus acidophilus. 
Gastroenterology. 1975;68:583-6.

24. Zweiban B, Cohen H, Chandrasoma P. Gastrointestinal involvement complicating Stevens-Johnson syndrome. Gastroenterology. 1986;91:469-674.

25. Le K, Ahmed A. Acute necrotizing esophagitis: case report and review of the literature. J La State Med Soc. 2007;159:330-8.

26. Orlando RC, Powell DW, Carney CN. Pathophysiology of acute acid injury in rabbit esophageal epithelium. J Clin Invest. 1981;68:286-93. doi: 10.1172/JCI110246.

27. De la Serna-Higuera C, Martinez J, Martin-Arribas M, Rodriquez-Gomez S, Perez-Villoria A, Betancourt A. Acute necrotizing esophagitis. Gastrointest Endosc. 2001;54(2):225 .

28. Moreto M, Ojembarrena E, Zaballa M, Tanago JG, Ibanez S. Idiopathic acute esophageal necrosis: not necessarily a terminal event. Endoscopy. 1993;25:534-8.
30. Husova L, Muckova K, Kroupa R, Dastych Jr M, Senkyrik M. Acute oesophageal necrosis. Cas Lek Cesk. 2005;144(1):48-52.

31. Swigart LL, Siekert RG, Hambley WC, Anson BJ. The esophageal arteries: an anatomic study of 150 specimens. Surg Gynecol Obstet. 1950;90:234-43.

32. Khalbuss WE, Marousis CG, Subramanyam M, Orlando RC. Effect of HCL on tranmembrane potentials and intracellular $\mathrm{pH}$ in rabbit esophageal epithelium. Gastroenterology. 1995;108:662-72. doi:10.1016/00165085(95)90437-9.

33. Singh, Dushyant, Reetu Singh, and Alexandra S. Laya Acute esophageal necrosis: a case series of five patients presenting with "Black esophagus". Indian J Gastroenterol. 2011;30(1):41-5.

34. Khan AM, Hundal R, Ramaswamy V, Korsten M, Dhuper S. Acute esophageal necrosis and liver pathology. 2004;10(16):2457-8. Available from: http://www.wjgnet. com/1007-9327/full/v10/i16/2457.htm. 